Recepción: 20 / 03 / 2019

Aceptación: 19 / 04 / 2019

Publicación: 05 / 06 / 2019

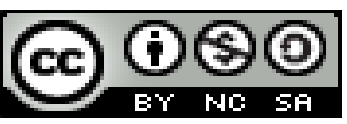

Ciencias de la educación

Artículo de Revisión

\title{
Incidencia de la Lectura Crítica en el Aprendizaje Significativo
}

Impact of Critical Reading on Significant Learning

Impacto da Leitura Crítica na Aprendizagem Significativa

Lenin B. Mendieta-Toledo ${ }^{\mathrm{I}}$ lenin.mendietat@ug.edu.ec

Oscar A. Garey-Atiencia II oscar.gareya@ug.edu.ec

Correspondencia: vladi.guerrero@hotmail.com

I. Licenciado en Ciencias de la Educación en la Especialidad de Educación Física; Magíster en Docencia Universitaria e Investigación Educativa; Universidad de Guayaquil; Guayaquil, Ecuador.

II. Universidad de Guayaquil; Guayaquil, Ecuador. 


\title{
Resumen
}

La lectura crítica es la base del pensamiento crítico y reflexivo, aquella que permite adquirir de mejor forma los aprendizajes en los estudiantes, el objetivo del estudio fue determinar la incidencia de la lectura crítica en el aprendizaje significativo de los estudiantes del séptimo grado del subnivel básica media. Fue un estudio experimental, bibliográfico, cuantitativo y descriptivo, se entrevistó a docentes y al director de la institución y se encuestó a los estudiantes, se realizó una prueba de lectura pre y pos programa. En la prueba de lectura, se evidenció que los estudiantes del grupo programa mejoraron en todos los criterios a evaluar, pasando de No Adquiere los Aprendizajes Requeridos a Domina los Aprendizajes Requeridos, mientras que los estudiantes del grupo control, no presentaron cambios en los dos momentos de la evaluación que fuesen significativos para señalar, se evidencia una mejoría en el rendimiento de los niños del grupo programa, en el consolidado de la calificación de la asignatura de lengua y literatura de los dos quimestres. La conclusión a la que se llegó fue que la intervención en estudiantes con un programa de aprendizaje de la lectura crítica, incide positivamente en el aprendizaje significativo de los estudiantes.

Palabras claves: Lectura Crítica; Aprendizaje Significativo; Programa de Lectura Crítica.

\begin{abstract}
Critical reading is the basis of critical and reflective thinking, which allows students to acquire better learning, the objective of the study was to determine the incidence of critical reading in meaningful learning of students in the seventh grade of the basic sublevel half. It was an experimental, bibliographic, quantitative and descriptive study, teachers and the director of the institution were interviewed and the students were surveyed, a reading test was carried out before and after the program. In the reading test, it was evidenced that the students of the group program improved in all the criteria to be evaluated, going from Not Acquire the Required Learning to Master the Required Learning, while the students of the control group, did not present changes in the two moments of the evaluation that were significant to indicate, there is evidence of an improvement in the performance of the children of the program group, in the consolidation of the qualification of the subject of language and literature of the two quimestres. The conclusion reached was that the intervention in students with a critical reading learning program, positively affects the significant learning of students.
\end{abstract}


Lenin B. Mendieta-Toledo; Oscar A. Garey-Atiencia.

Keys words: Critical Reading; Significant learning; Critical Reading Program.

\section{Resumo.}

A leitura crítica é a base do pensamento crítico e reflexivo, que permite aos alunos adquirir uma melhor aprendizagem, o objetivo do estudo foi determinar a incidência de leitura crítica na aprendizagem significativa dos alunos na sétima série do subnível básico médio Tratou-se de um estudo experimental, bibliográfico, quantitativo e descritivo, os professores e o diretor da instituição foram entrevistados e os alunos foram pesquisados, realizou-se um teste de leitura antes e após o programa. No teste de leitura, constatou-se que os alunos do grupo de programa melhoraram em todos os critérios a serem avaliados, passando de Não Adquirir o Aprendizado Exigido a Mestrar a Aprendizagem Requerida, enquanto os alunos do grupo controle, não apresentaram mudanças nos dois momentos. da avaliação que foram significativos para indicar, há evidências de uma melhora no desempenho das crianças do grupo do programa, na consolidação da qualificação da disciplina de linguagem e literatura dos dois quimestres. A conclusão alcançada foi que a intervenção em alunos com um programa de aprendizagem de leitura crítica, afeta positivamente a aprendizagem significativa dos alunos.

Palavras chaves: Leitura Crítica; Aprendizagem significativa; Programa de Leitura Crítica.

\section{Introducción.}

Leer es el lenguaje utilizado por los seres humanos, constituyéndose en parte inherente a estos, (Acosta \& García , 2012; Alvarado, 2013) señalan que la lectura es la puerta que nos traslada a un mundo de riqueza infinita de conocimientos, resaltan que es una tarea intelectual que permite al cerebro activarse, ordenando ideas, relacionando conceptos y provocando reacciones y opiniones de parte del sujeto lector, estas reacciones provocan conexiones neuronales que a su vez permiten el pensamiento crítico y de esta forma, el ejercicio de funciones como memoria, concentración y atención. A pesar de la importancia de la lectura como base del aprendizaje, en la actualidad se presentan una serie de dispositivos que resultan distractores para el niño y obstáculo para el docente en su labor por enseñar. 
La lectura, es el acto de comprender lo escrito, de comprender las ideas que están detrás de las palabras y que forman oraciones, frases, párrafos y textos. (Solé, 1987a) señala que lees es "un proceso de interacción entre el lector y el texto, proceso mediante el cual el primero intenta satisfacer (obtener información pertinente para) los objetivos que guían su lectura" (Citado en Solé, 2009, p. 17). Para que se pueda llamar lectura el sujeto debe tener la competencia lectora y es así que la (oecd, 2006b, p. 48) indica que lectura es "La capacidad de comprender, utilizar y analizar textos escritos para alcanzar los objetivos del lector, desarrollar sus conocimientos y posibilidades y participar en la sociedad" (Citado en Pérez \& Roa , 2014, p. 25).

La lectura se genera cuando existe el sujeto lector, que desde sus requisitos previos atribuye sentido y significado al texto, para que exista este sujeto, alguién debió enseñarle el acto de leer, es una situación de intervención dialéctica-pedagógica, en donde, el aprendiz, necesita los insumos pedagógicodidácticos que el profesor le brinda para que se convierta en lector, para que exista la lectura es preciso por supuesto, que exista “el sujeto que procesa y le aporta sus conocimientos, experiencias y esquemas previos" (Solé, 2009, p. 14).

Ramírez (2009) manifiesta que leer y lectura desde la fenomenología o como constructo conceptual, pueden ser a la vez simples o complejas, dependiendo del sujeto que analice este verbo y sustantivo (respectivamente) que la lectura es la decodificación y comprensión de las palabras escritas, pero que sin embargo, esta es una tarea que parece simple y, que no se enmarca en la complejidad que esta conlleva, pues al sustraerla de las condiciones subjetivas, culturales y socio-históricas contenidas en las representaciones simbólicas de esas prácticas sociales de la lectura del objeto de conocimiento escrito, se desfavorece el análisis cabal que fundamente y explique el fenómeno y por supuesto, con ello, se limita a la episteme del objeto construido. 
Remolina (2013) sobre la definición de lectura que hace la Real Academia Española como la "acción de leer", manifiesta, leer se encuentra como un verbo transitivo que significa comprender o interpretar un texto de una u otra forma, dependiendo de los requisitos previos con los que cuente el sujeto lector. El acto de leer posee categorías conceptuales como la operacionalidad cognitiva, el código representacional, y, la modalidad interpretativa.

La lectura crítica es la técnica que permite desvelar las ideas del autor del texto que, a pesar de no estar expresadas en forma extrínseca en un texto, se encuentran en él. Para esto, se precisa de una lectura analítica, reflexiva y por supuesto activa del sujeto lector, el cual deberá ser crítico. Lipman (1997), señala que "es necesario propiciar el ejercicio del análisis crítico desde los primeros niveles educativos. Es por ello que Newman (1990) indica que hay que enseñar en base a la lectura crítica ya que esta condice al cultivo del pensamiento crítico o de orden superior, el mismo, generará en el sujeto solvencia a la hora de resolver situaciones de conflicto, lograr independencia de juicio en la medida que éstas desafíen creencias o paradigmas establecidos en los requisitos previos del lector (Citados en Díaz, Bar, \& Ortíz, 2015, p. 141).

Un lector crítico, es aquel comprende autónomamente el propósito lingüístico, las intenciones pragmáticas y los puntos de vista particulares que subyacen a los discursos que le rodean, es aquel que tiene consciencia del contexto desde el que se ha generado la narrativa; logra deconstruir los discursos del autor sin dislocar la escencia del mensaje, maneteniendo su postura viculada polífonica o intertextualmente con el discurso original; sabe que dispositivos lingüísticos utilizar para conseguir representar desde un discurso autónomo su opinión a través de esos segundos discursos.

(Ballester, 2002; Rodríguez, 2008; Díaz, 2010) coinciden en que "el aprendizaje es entendido como la reesctructuración activa de las percepciones, ideas, conceptos y esquemas que el aprendiz posee en 
su estructura cognitiva. El aprendizaje era concebido de forma conceptual y no se llegaba a determinar la escencia del verdadero aprendizaje, hasta que en la decada de los años sesenta del siglo próximo pasado, Ausubel utilizó el termino aprendizaje significativo, el mismo que es entendido como el producto de la interacción entre un material o una información nueva y la estructura congnitiva preexistente, constituyéndose los significados en una construcción muy particular del sujeto que aprende, el cual realiza ese aprendizaje de forma intima, debido a que el asimilar un nuevo objeto implica que el sujeto deforme el objeto y lo deconstruya. Ausubel (1973) manifestó que "el aprendizaje significativo es la vía por la que las personas asimilan la cultura que les rodea" (Citado por Pozo, 2006, p. 215)

Diversos estudios (Mosquera, 2017; Nivelo \& Quijije, 2017; Cornejo \& Cuzco, 2017) coinciden en que existe una marcada incidencia de la lectura crítica en el aprendizaje significativo de los estudiantes. Roca (2013) concluyó que "La lectura crítica como competencia genérica, debe estar presente en todo proceso formativo, por su utilidad personal, profesional y por su capacidad de transferencia a nuevas y diferentes situaciones" (p. 23); o a la inversa, como señala Mosquera (2017), la deficiente lectura crítica es causa del deficiente desarrollo de nuevos aprendizajes significativos en los estudiantes.

El problema que se desarrolló en estudiantes que presentaban dificultad en la lectura, dificultad para comprender y analizar textos y por tanto deficiencia en el razonamiento e interpretación de las lecturas, esto en la Escuela "Medardo Alfaro" en la provincia del Guayas

El objetivo del estudio fue determinar la incidencia de la lectura crítica en el aprendizaje significativo de los estudiantes del subnivel básico medio.

\section{Materiales y métodos.}


Fue un estudio experimental mediante una prueba controlada aleatorizada (White, Sabarwal, \& de Hoop, 2014), para determinar la incidencia de la lectura crítica en el aprendizaje significativo, fue una investigación de corte bibliográfica, cuantitativa y descriptiva, se utilizaron las técnicas de la entrevista para docentes y director de la institución y encuesta a estudiantes, con el instrumento del cuestionario para cada uno de estos, se utilizó la escala de Likert con los parámetros de: siempre, frecuentemente, a veces, nunca para la encuesta; se realizó una prueba de lectura pre y pos programa a los estudiantes, para su evaluación se diseñó una rúbrica que mantenía los criterios del Ministerio de Educación del Ecuador, para ese nivel educativo.

El estudio se realizó entre septiembre del 2017 y enero del 2018, se solicitó el consentimiento informado por escrito de los padres de familia para que los estudiantes participen en el programa de lectura, se establecieron dos grupos de una muestra de 40 estudiantes de un total de 44 , se tomó en cuenta un criterio de inclusión (no posean discapacidad intelectual), los cuatro estudiantes que no participaron del estudio se debieron a que pertenecían al grupo de exclusión. Se trabajó con un grupo programa (1) de 20 estudiantes y un grupo control (0) con 20 estudiantes, se realizó una prueba de lectura crítica antes de iniciar el programa y al otra al finalizar el mismo (semana 0 y semana 13 , no se correspondían al programa), además, se evaluó el consolidado de calificaciones en lengua y literatura de los estudiantes del quimestre anterior al estudio (septiembre), se contrastó con el consolidado de calificaciones del quimestre final (febrero 2018).

\section{Diagrama de flujo.}

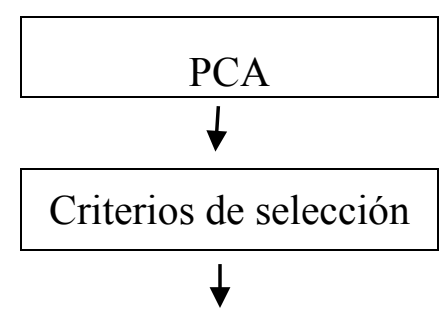




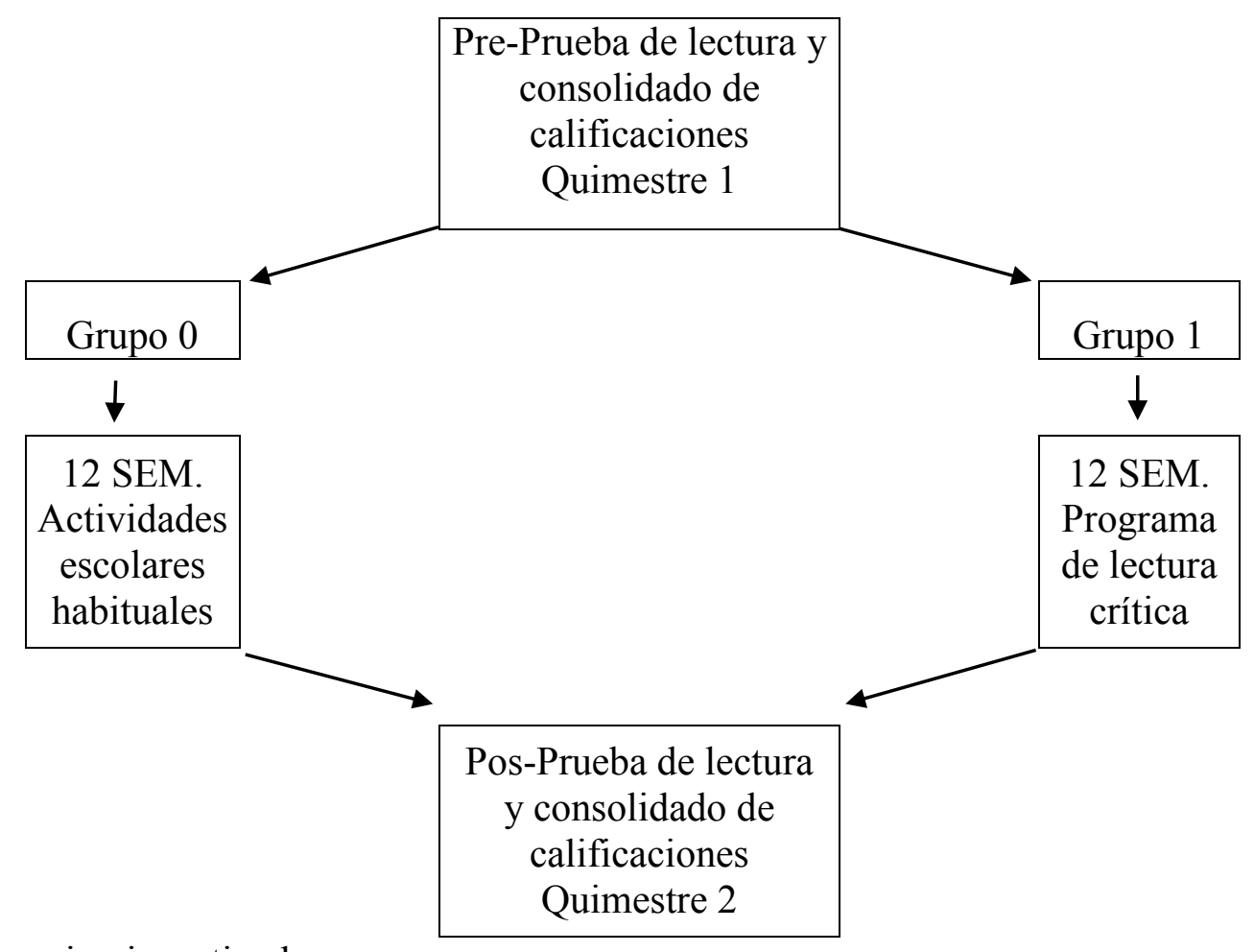

Diseño: equipo investigador

${ }^{1}$ Investigador principal, experto en lectura crítica en el nivel educativo básico; segundo investigador, experto en lengua y literatura con más de 40 años de docencia en todos los niveles educativos.

El grupo control (0) trabajó normalmente en sus clases diarias, se les recomendó que una buena lectura ayuda a mejorar en la adquisición de aprendizajes y en el rendimiento académico, el grupo programa, participó de un programa de lectura crítica de una duración de 12 semanas, tres días a la semana de forma alternada, con una duración de una hora cada sesión, el programa se basó en una guía pedagógica de lectura crítica elaborada por el equipo de investigación ${ }^{1}$.

Se realizaron 36 sesiones en donde se establecieron objetivos para cada periodo y sesión, se diseñaron 6 periodos (periodo estuvo constituido por seis sesiones).

Planificación de un periodo de trabajo del programa.

\begin{tabular}{|c|c|c|c|}
\hline $\begin{array}{c}\text { Título de la } \\
\text { Unidad }\end{array}$ & Objetivo & $\begin{array}{c}\text { Destrezas con } \\
\text { criterio de } \\
\text { desempeño a ser } \\
\text { desarrolladas }\end{array}$ & $\begin{array}{c}\text { Indicadores esenciales de } \\
\text { evaluación }\end{array}$ \\
\hline
\end{tabular}

\footnotetext{
${ }^{1}$ Investigador principal, experto en lectura crítica en el nivel educativo básico; segundo investigador, experto en lengua y literatura con más de 40 años de docencia en todos los niveles educativos.
} 


\begin{tabular}{|c|c|c|c|}
\hline $\begin{array}{l}\text { Lecturas } \\
\text { soñadoras }\end{array}$ & $\begin{array}{l}\text { Leer de manera autónoma } \\
\text { textos no literarios, con } \\
\text { fines de recreación, } \\
\text { información y aprendizaje, } \\
\text { y utilizar estrategias } \\
\text { cognitivas de comprensión } \\
\text { de acuerdo al tipo de texto. }\end{array}$ & $\begin{array}{l}\text { Escuchar y leer } \\
\text { diversos géneros } \\
\text { literarios } \\
\text { (privilegiando textos } \\
\text { ecuatorianos, } \\
\text { populares y de autor), } \\
\text { para potenciar la } \\
\text { imaginación, la } \\
\text { curiosidad y la } \\
\text { memoria. }\end{array}$ & $\begin{array}{l}\text { Construye criterios, opiniones y } \\
\text { emite juicios acerca del } \\
\text { contenido de un texto, al } \\
\text { distinguir realidad y ficción, } \\
\text { hechos, datos y opiniones, y } \\
\text { desarrolla estrategias cognitivas } \\
\text { como lectura de para textos, } \\
\text { establecimiento del propósito } \\
\text { de lectura, relectura, relectura } \\
\text { selectiva y parafraseo, para } \\
\text { autorregular la comprensión. } \\
\text { (J.4., I.3.) }\end{array}$ \\
\hline $\begin{array}{l}\text { Estrategias } \\
\text { metodológicas }\end{array}$ & $\begin{array}{l}\text { Prelectura: Entregar la } \\
\text { imagen en fotocopia de la } \\
\text { lectura para que la } \\
\text { coloreen, para que } \\
\text { inventen un título y una } \\
\text { historia relacionada con el } \\
\text { dibujo. Esta lectura literal } \\
\text { motiva la estructura } \\
\text { cognitiva para que el } \\
\text { estudiante participe dentro } \\
\text { y fuera del aula en } \\
\text { reconocer, identificar y } \\
\text { comunicarse a nivel social } \\
\text { y cultural }\end{array}$ & $\begin{array}{l}\text { Lectura: Lectura "El } \\
\text { triste sueño del } \\
\text { mono"; Leer en voz } \\
\text { alta de manera coral.; } \\
\text { Leer de manera } \\
\text { individual; } \\
\text { Comprender las ideas } \\
\text { que no estén escritas } \\
\text { expresamente y las } \\
\text { que están explícitas }\end{array}$ & $\begin{array}{l}\text { Postlectura: Realiza y responde } \\
\text { preguntas del texto que se } \\
\text { refieran a lo literal y a lo que } \\
\text { debe deducirse; Comprende el } \\
\text { desarrollo de las ideas de la } \\
\text { lectura. Verifica lo que se } \\
\text { predijo; Busca la diferencia } \\
\text { entre las figuras; Parafrasea } \\
\text { información y realizar un } \\
\text { resumen }\end{array}$ \\
\hline Recursos & Texto del estudiante & Cuaderno de trabajo & $\begin{array}{l}\text { Fábulas de Esopo; Carteles con } \\
\text { moralejas }\end{array}$ \\
\hline $\begin{array}{l}\text { Indicadores de } \\
\text { logro }\end{array}$ & $\begin{array}{l}\text { Comprende las ideas } \\
\text { explícitas de las fábulas }\end{array}$ & $\begin{array}{l}\text { Realiza } \\
\text { comparaciones entre } \\
\text { lo que dice la fábula } \\
\text { El triste sueño del } \\
\text { mono y la realidad. }\end{array}$ & $\begin{array}{l}\text { Reflexiona sobre el mensaje de } \\
\text { las fábulas }\end{array}$ \\
\hline $\begin{array}{l}\text { Evaluación/ } \\
\text { Técnicas / } \\
\text { instrumentos }\end{array}$ & Técnica: Observación & Instrumentos: & $\begin{array}{l}\text { Ejercicios y prácticas en clase y } \\
\text { contestar preguntas }\end{array}$ \\
\hline
\end{tabular}

Diseño: equipo de investigación

\section{Resultados.}


Cuadro $N^{o} 1$. Promedio del consolidado de calificaciones en lengua y literatura Grupo (0) y

\section{Grupo (1) $Q 1$ y $Q^{2}$}

\begin{tabular}{|c|c|c|c|c|c|c|c|c|c|c|c|}
\hline Asignatura & \multicolumn{2}{|c|}{ 1er Parcial } & \multicolumn{2}{|c|}{ 2do parcial } & \multicolumn{2}{|c|}{ 3er parcial } & $\begin{array}{c}\text { Prom } \\
\text { tres } \\
\text { Parciales }\end{array}$ & $\begin{array}{c}\text { Prom } \\
\mathbf{8 0} \%\end{array}$ & $\begin{array}{c}\text { Examen } \\
\mathbf{2 0 \%}\end{array}$ & $\begin{array}{c}\text { Nota } \\
\text { Q1 }\end{array}$ & Cualitativa \\
\hline G0 & Prom & Cual & Prom & Cual & Prom & Cual & & & & &
\end{tabular}

Diseño: equipo de investigación

Fuente: sujetos de estudio

Gráfico $N^{o} 1$. Promedio del consolidado de calificaciones en lengua y literatura Grupo (0) y Grupo

(1) $Q 1$ y $Q^{2}$ 


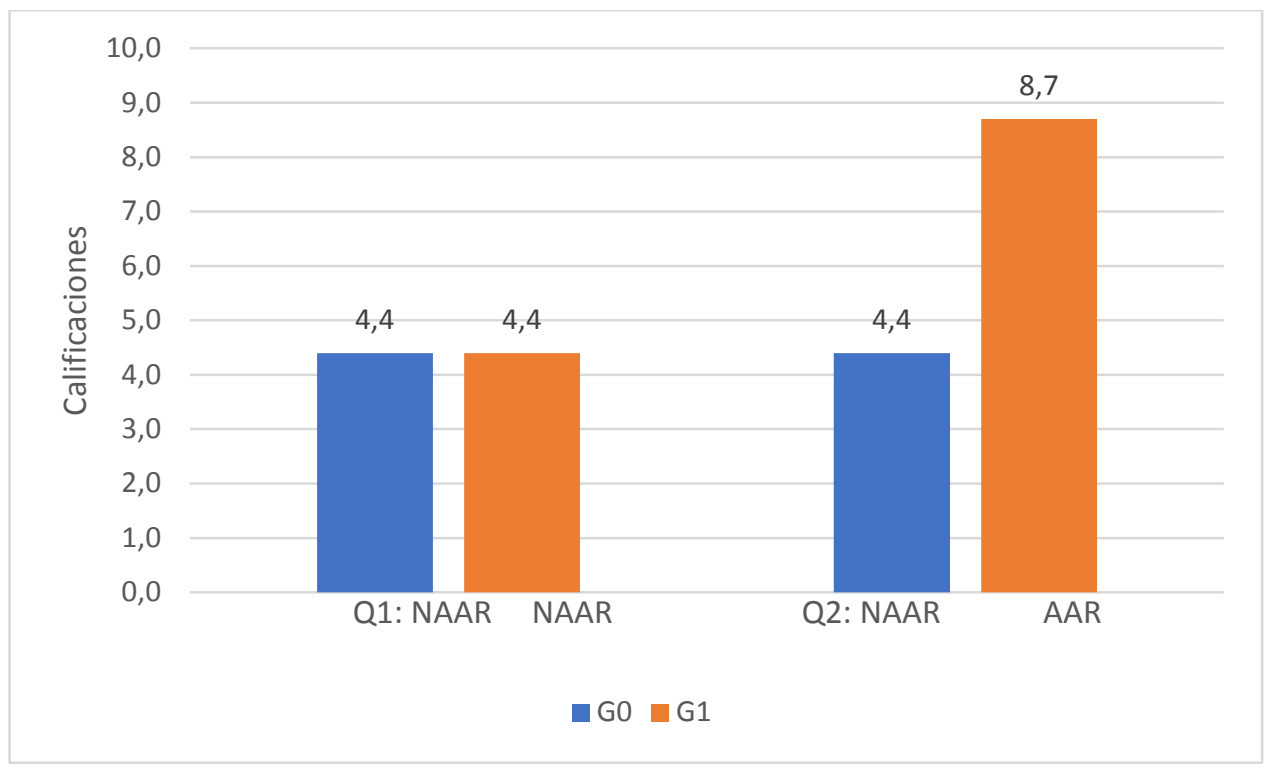

Diseño: equipo de investigación

Fuente: sujetos de estudio

\section{Cuadro $N^{\circ} 2$. Encuesta a estudiantes}

\begin{tabular}{|c|c|c|c|c|c|}
\hline \multicolumn{2}{|r|}{ Preguntas } & Siempre & Frecuente & A veces & Nunca \\
\hline 1 & $\begin{array}{l}\text { ¿Cuándo lees en clase comprendes y te } \\
\text { interesas sobre lo que aprendes? }\end{array}$ & 2 & 4 & 18 & 16 \\
\hline 2 & $\begin{array}{l}\text { ¿Sientes gusto al leer sobre algún tema dado } \\
\text { por el docente? }\end{array}$ & 5 & 6 & 14 & 15 \\
\hline 3 & $\begin{array}{l}\text { ¿El docente realiza juegos acertijos, } \\
\text { adivinanzas (le motiva) antes de iniciar una } \\
\text { lectura? }\end{array}$ & 4 & 4 & 21 & 11 \\
\hline 4 & $\begin{array}{l}\text { ¿Las actividades que realizan los docentes } \\
\text { ayudan aprender de forma interesante? }\end{array}$ & 7 & 14 & 7 & 12 \\
\hline 5 & $\begin{array}{l}\text { ¿Identificas con facilidad los personajes y los } \\
\text { escenarios del tema de la lectura en clases? }\end{array}$ & 2 & 2 & 12 & 24 \\
\hline 6 & $\begin{array}{l}\text { ¿Comprendes y expresas con tus propias } \\
\text { palabras de lo que se trata en la lectura? }\end{array}$ & 2 & 8 & 11 & 19 \\
\hline 7 & $\begin{array}{l}\text { ¿Reconoces reglas de lectura como la } \\
\text { pronunciación y signos de puntuación para una } \\
\text { buena comprensión lectora? }\end{array}$ & 1 & 3 & 16 & 20 \\
\hline 8 & $\begin{array}{l}\text { ¿Diferencias entre los tipos de lectura que } \\
\text { realizas en clase y horas libres? }\end{array}$ & 1 & 3 & 17 & 19 \\
\hline 9 & $\begin{array}{l}\text { ¿El docente te guía en la lectura para ayudarte a } \\
\text { pensar rápido? }\end{array}$ & 3 & 4 & 18 & 15 \\
\hline
\end{tabular}




\section{Gráfico $N^{o} 2$. Encuesta a estudiantes}

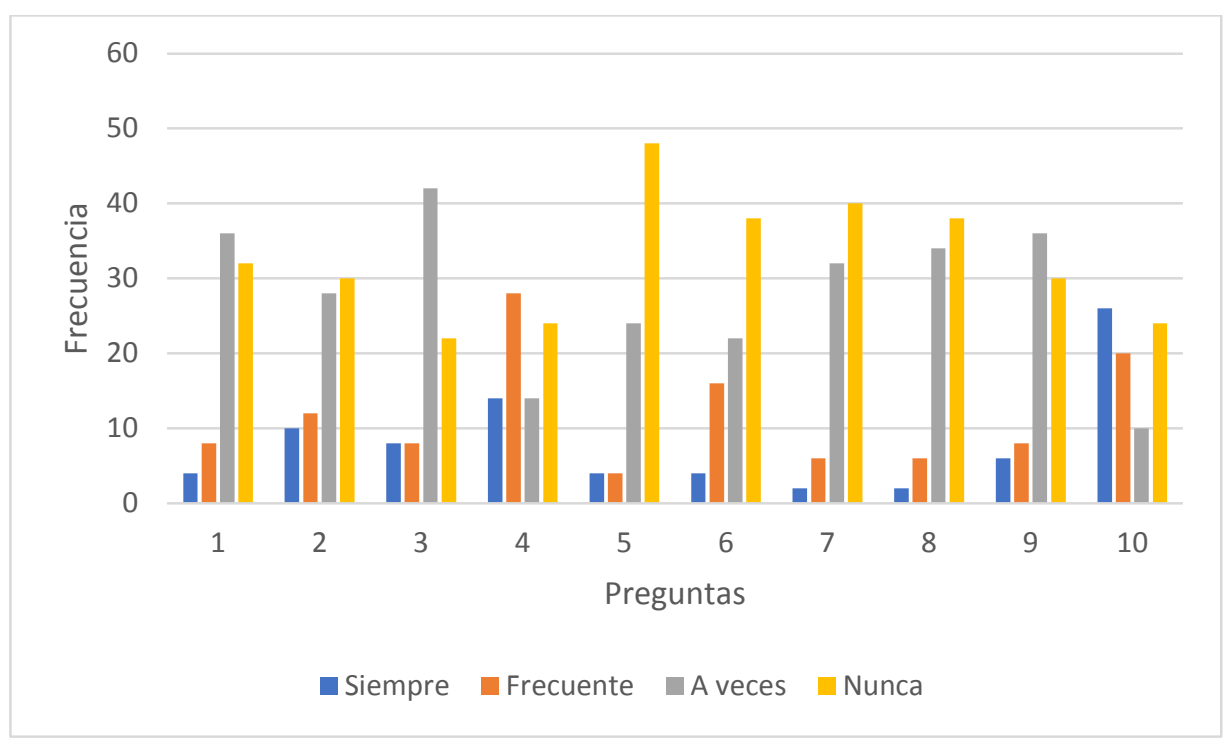

Diseño: equipo de investigación

Fuente: sujetos de estudio

Cuadro $N^{o} 3$. Destrezas con criterio de desempeño evaluadas en la prueba de lectura 
Lenin B. Mendieta-Toledo; Oscar A. Garey-Atiencia.

\begin{tabular}{|c|c|c|c|c|c|c|c|c|c|c|c|c|c|c|c|c|c|c|c|c|}
\hline \multirow{3}{*}{\begin{tabular}{|c|} 
Grupos \\
Momento de evalución
\end{tabular}} & \multicolumn{20}{|c|}{ PARÁMETROS } \\
\hline & \multicolumn{2}{|c|}{ G0 } & \multicolumn{2}{|c|}{ G1 } & \multicolumn{2}{|c|}{ G0 } & \multicolumn{2}{|c|}{ G1 } & \multicolumn{2}{|c|}{ G0 } & \multicolumn{2}{|c|}{ G1 } & \multicolumn{2}{|c|}{ G0 } & \multicolumn{2}{|c|}{ Gl } & \multicolumn{2}{|c|}{ G0 } & \multicolumn{2}{|c|}{ G1 } \\
\hline & PRE EV & POS EV & PRE EV & POS EV & PRE EV & POS EV & PRE EV & POS EV & PRE EV & POS EV & PRE EV & POS EV & PRE EV & POS EV & PRE EV & POS EV & PRE EV & POS EV & PRE EV & POS EV \\
\hline Indicadores & SAR & SAR & SAR & SAR & DAR & DAR & DAR & DAR & AAR & AAR & AAR & AAR & EPAR & EPAR & EPAR & \begin{tabular}{|l|} 
EPAR \\
\end{tabular} & NAAR & NAAR & NAAR & NAAR \\
\hline $\begin{array}{l}\text { Lee de manera autónoma textos no } \\
\text { literarios, con fines de recreación, } \\
\text { información y aprendizaje. }\end{array}$ & 0 & 0 & 0 & 19 & 0 & 0 & 1 & 0 & 0 & 2 & 0 & 0 & 2 & 2 & 1 & 1 & 18 & 16 & 18 & 0 \\
\hline $\begin{array}{l}\text { Utiliza estrategias cognitivas de } \\
\text { comprensión de acuerdo al tipo de } \\
\text { texto }\end{array}$ & 0 & 0 & 0 & 17 & 0 & 0 & 1 & 3 & 0 & 2 & 0 & 0 & 2 & 2 & 1 & 0 & 18 & 16 & 18 & 0 \\
\hline $\begin{array}{l}\text { Comprende el desarrollo de las ideas } \\
\text { de la lectura. }\end{array}$ & 0 & 0 & 0 & 16 & 0 & 0 & 0 & 4 & 0 & 2 & 1 & 0 & 2 & 2 & 1 & 0 & 18 & 16 & 18 & 0 \\
\hline \begin{tabular}{|l|} 
Verifica lo que se predijo; Busca la \\
diferencia entre las figuras; Parafrasea \\
información y realiza un resumen
\end{tabular} & 0 & 0 & 0 & 18 & 0 & 0 & 1 & 2 & 0 & 2 & 0 & 0 & 2 & 2 & 1 & 0 & 18 & 16 & 18 & 0 \\
\hline $\begin{array}{l}\text { Desarrolla estrategias cognitivas como } \\
\text { lectura de paratextos, establecimiento } \\
\text { del propósito de lectura, relectura, } \\
\text { relectura selectiva y autorregula la } \\
\text { comprensión }\end{array}$ & 0 & 0 & 0 & 19 & 0 & 0 & 1 & 0 & 0 & 2 & 0 & 0 & 2 & 2 & 1 & 1 & 18 & 16 & 18 & 0 \\
\hline $\begin{array}{l}\text { Realiza y responde preguntas del texto } \\
\text { que se refieran a lo literal y a lo que } \\
\text { debe deducirse }\end{array}$ & 0 & 0 & 0 & 17 & 0 & 0 & 1 & 3 & 0 & 2 & 0 & 0 & 2 & 2 & 1 & 0 & 18 & 16 & 18 & 0 \\
\hline $\begin{array}{l}\text { Construye criterios, opiniones y emite } \\
\text { juicios acerca del contenido de un } \\
\text { texto, al distinguir realidad y ficción, } \\
\text { hechos, datos y opiniones }\end{array}$ & 0 & 0 & 0 & 16 & 0 & 0 & 0 & 4 & 0 & 2 & 1 & 0 & 2 & 2 & 1 & 0 & 18 & 16 & 18 & 0 \\
\hline
\end{tabular}

Diseño: equipo de investigación

Fuente: sujetos de estudio

\section{Indicadores.}

Destrezas con criterio de desempeño evaluadas en la prueba de lectura 
Gráfico $N^{o 3}$. Lee de manera autónoma textos no literarios, con fines de recreación, información y

\section{aprendizaje}

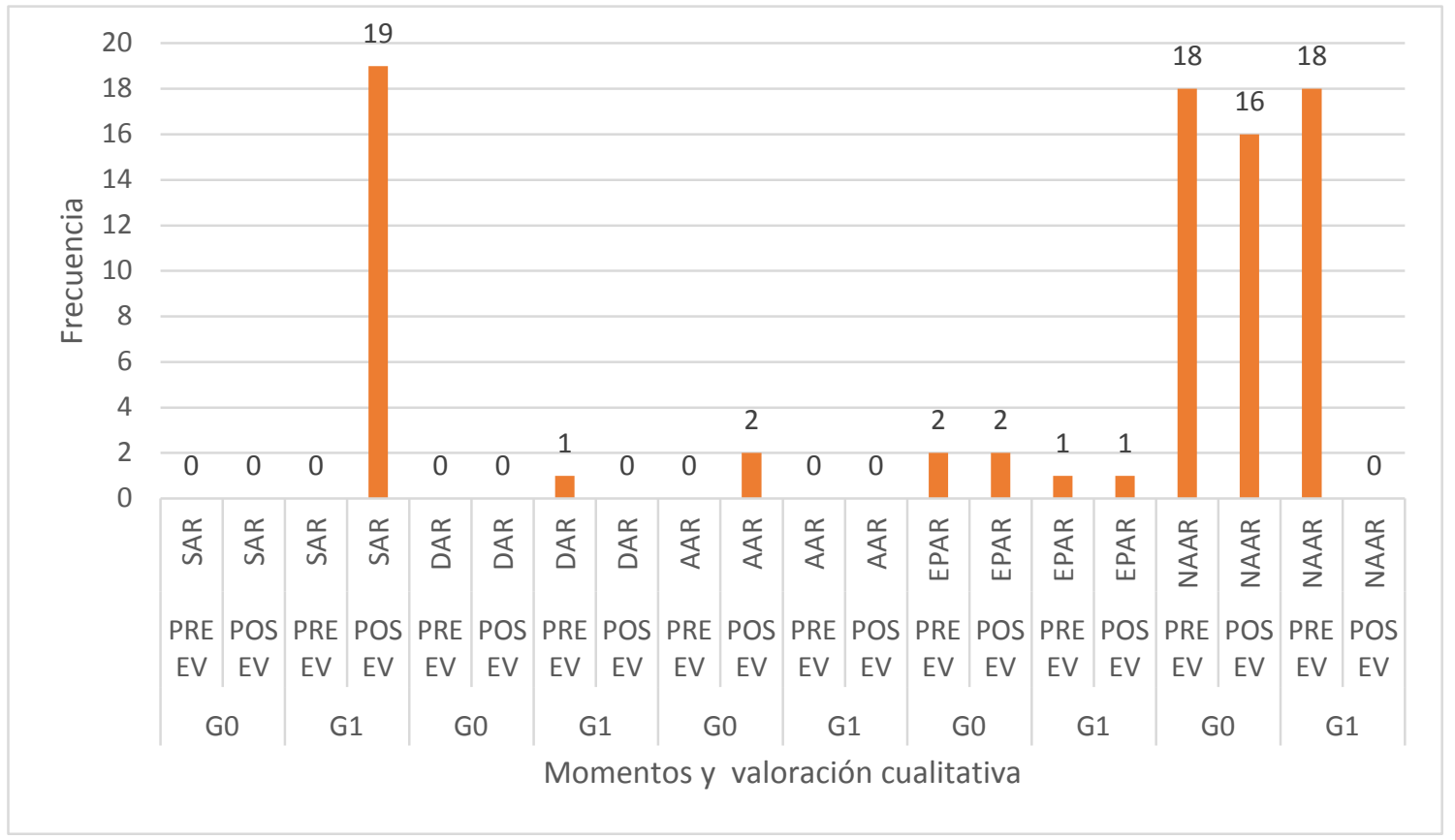

Diseño: equipo de investigación

Fuente: sujetos de estudio 
Lenin B. Mendieta-Toledo; Oscar A. Garey-Atiencia.

Gráfico $N^{o}$ 4. Utiliza estrategias cognitivas de comprensión de acuerdo al tipo de texto

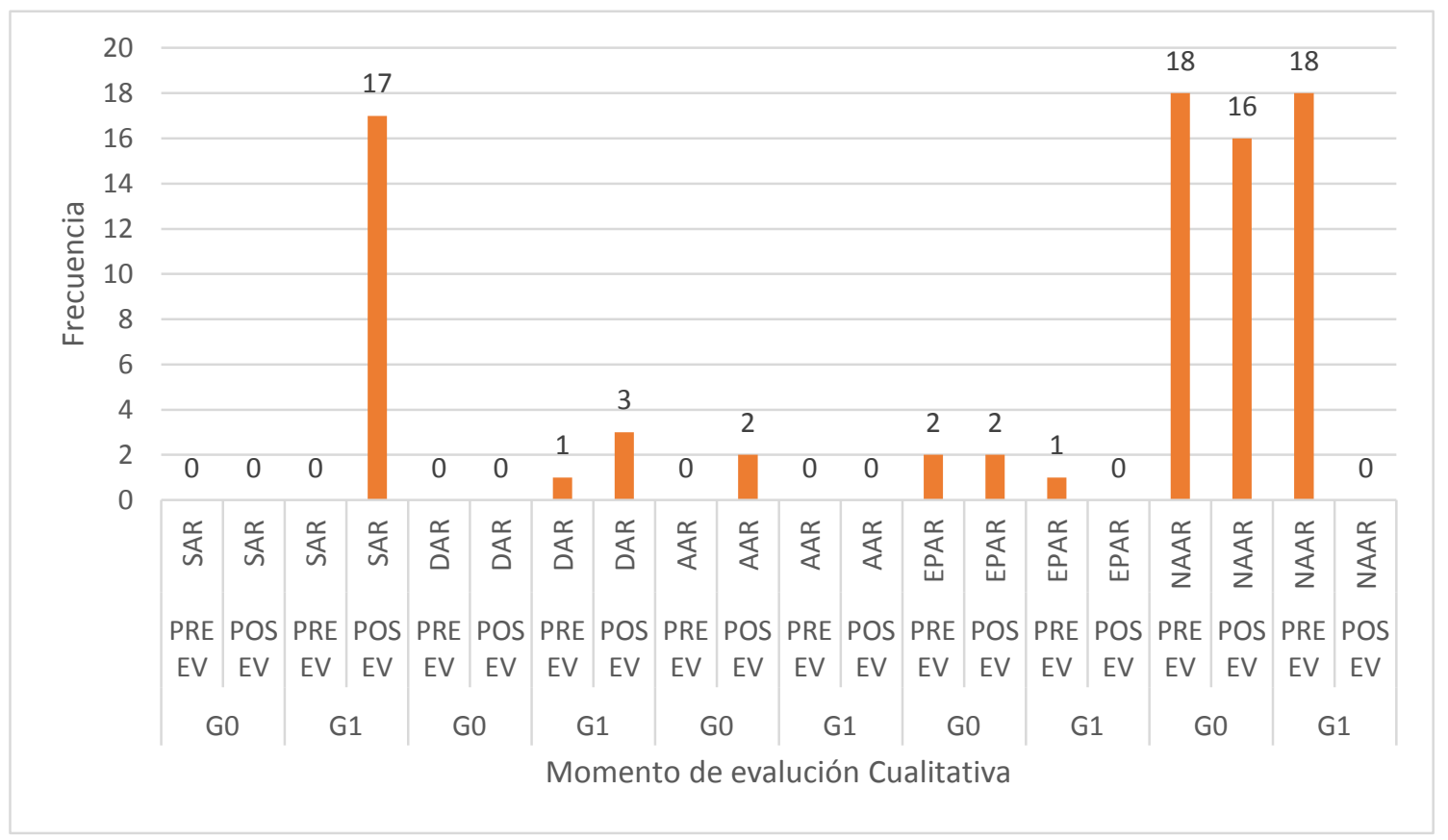

Diseño: equipo de investigación

Fuente: sujetos de estudio 


\section{Gráfico $N^{o} 5$. Comprende el desarrollo de las ideas de la lectura}

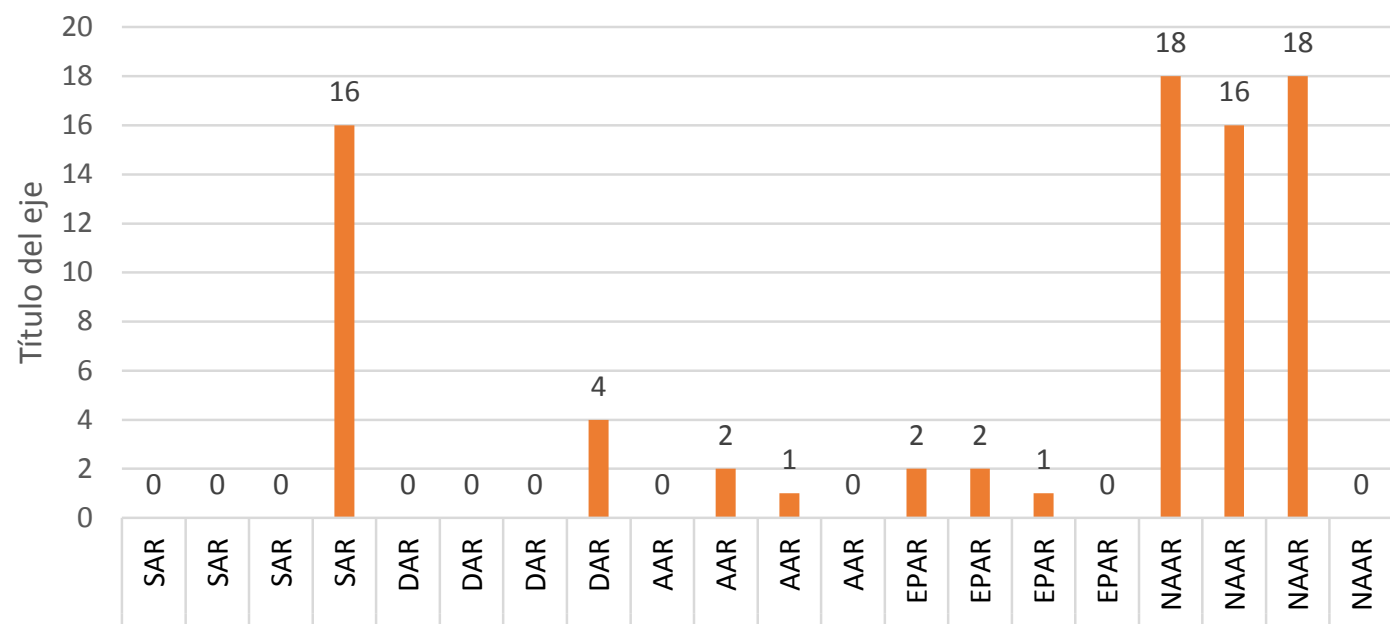

PRE POS PRE POS PRE POS PRE POS PRE POS PRE POS PRE POS PRE POS PRE POS PRE POS

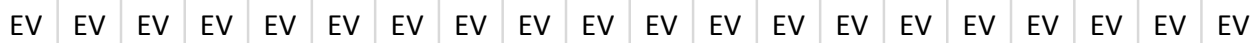

\begin{tabular}{|l|l|l|l|l|l|l|l|l|l|}
$\mathrm{G} 0$ & $\mathrm{G1}$ & $\mathrm{G} 0$ & $\mathrm{G} 1$ & $\mathrm{G} 0$ & $\mathrm{G} 1$ & $\mathrm{G} 0$ & $\mathrm{G} 1$ & $\mathrm{G} 0$ & $\mathrm{G} 1$ \\
\hline
\end{tabular}

Momento y evaluación cualitativa

Diseño: equipo de investigación

Fuente: sujetos de estudio

Gráfico $N^{o}$ 6. Verifica lo que se predijo, busca la diferencia entre las figuras, parafrasea información y realiza un resumen

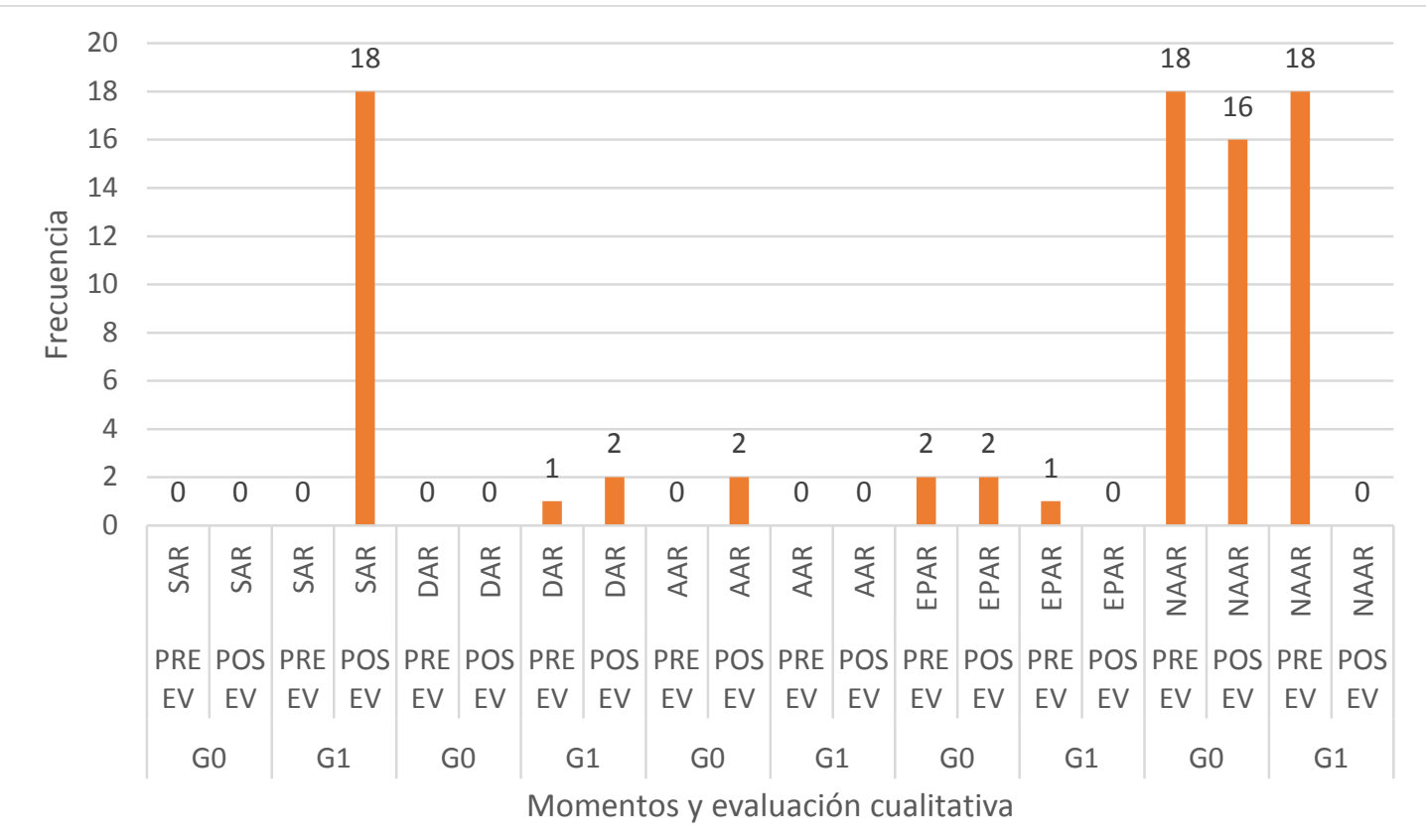

Diseño: equipo de investigación

Fuente: sujetos de estudio 
Gráfico $N^{o}$ 7. Desarrolla estrategias cognitivas como lectura de para textos, establecimiento del propósito de lectura, relectura, relectura selectiva y autorregula la comprensión

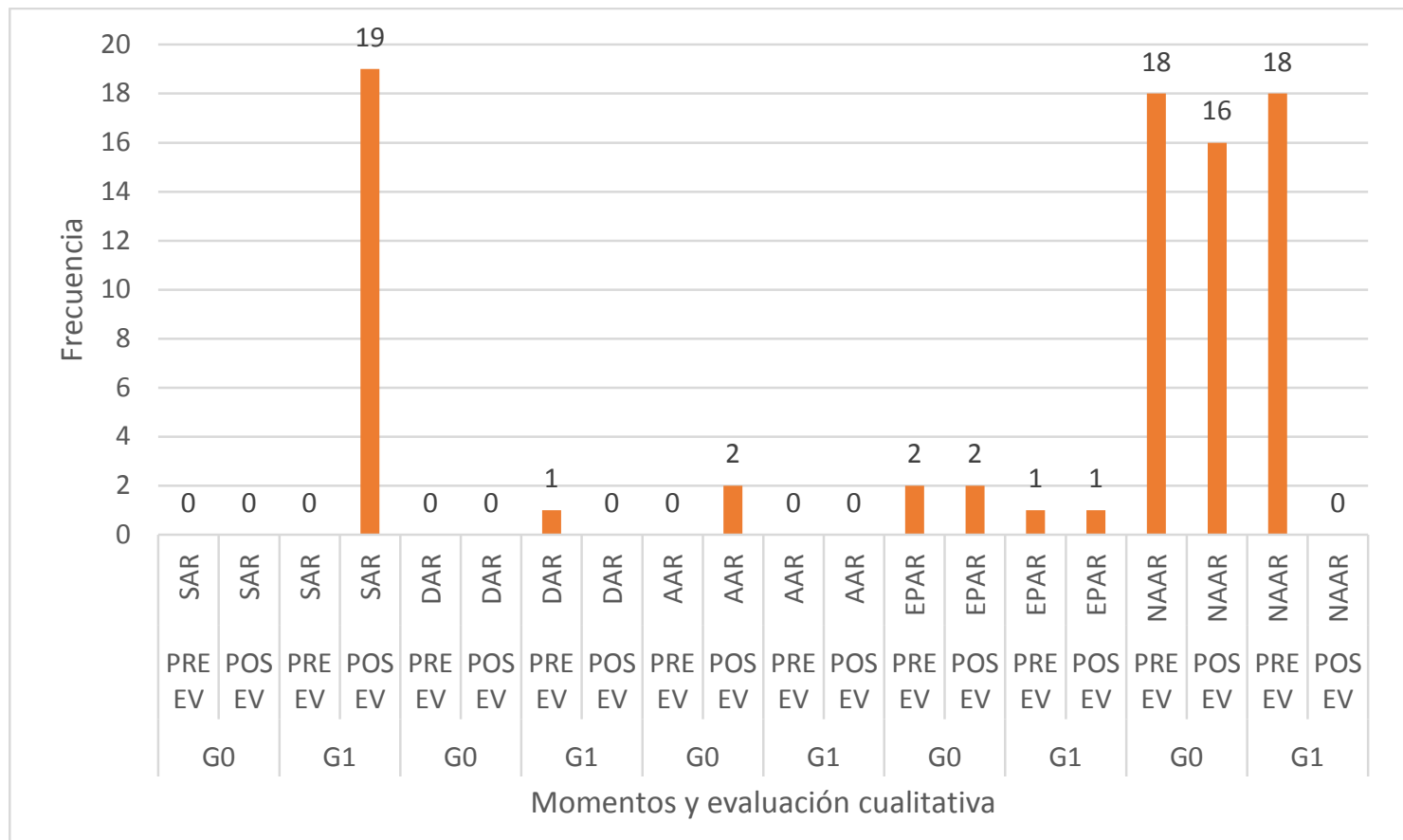

Diseño: equipo de investigación

Fuente: sujetos de estudio

Gráfico $N^{o} 8$. Realiza y responde preguntas del texto que se refieran a lo literal y a lo que debe deducirse

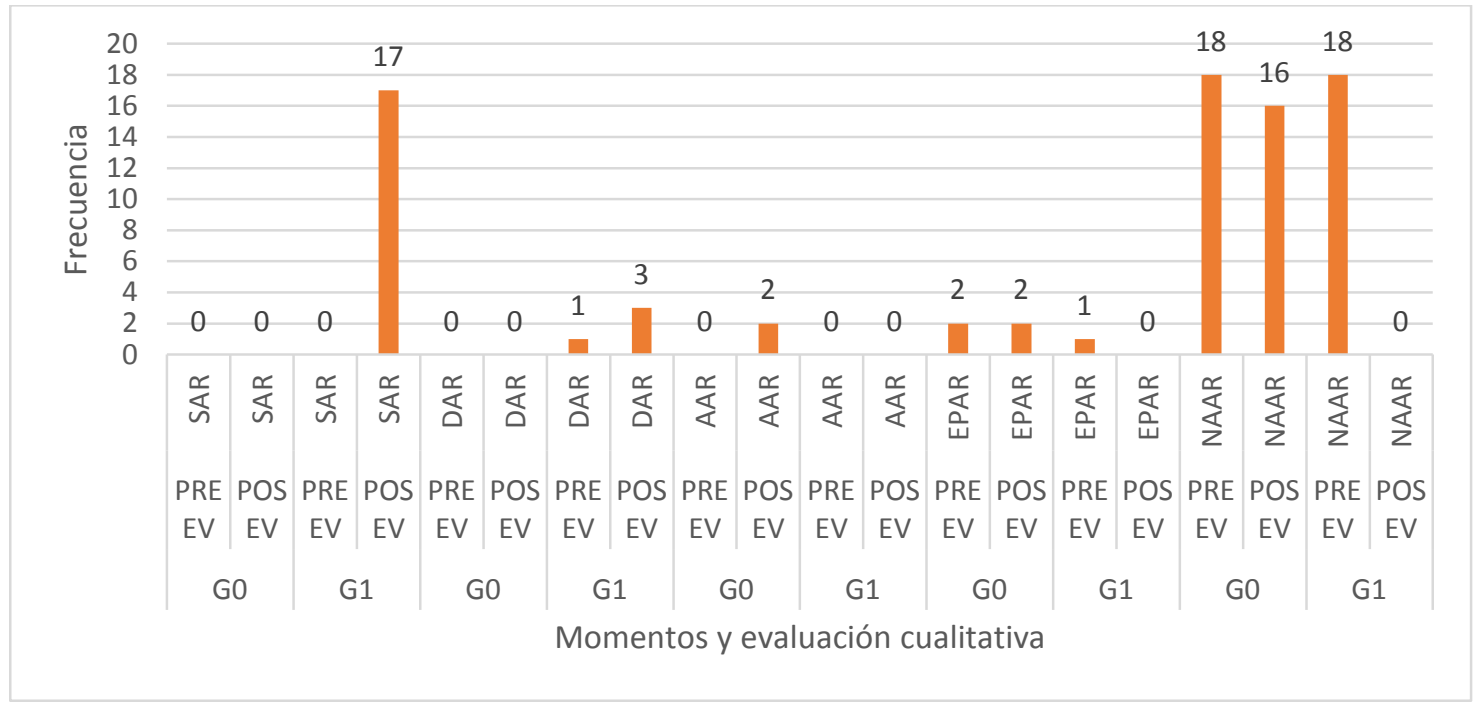

Diseño: equipo de investigación

Fuente: sujetos de estudio 
Gráfico $N^{o}$ 9. Construye criterios, opiniones y emite juicios acerca del contenido de un texto, al distinguir realidad y ficción, hechos, datos y opiniones

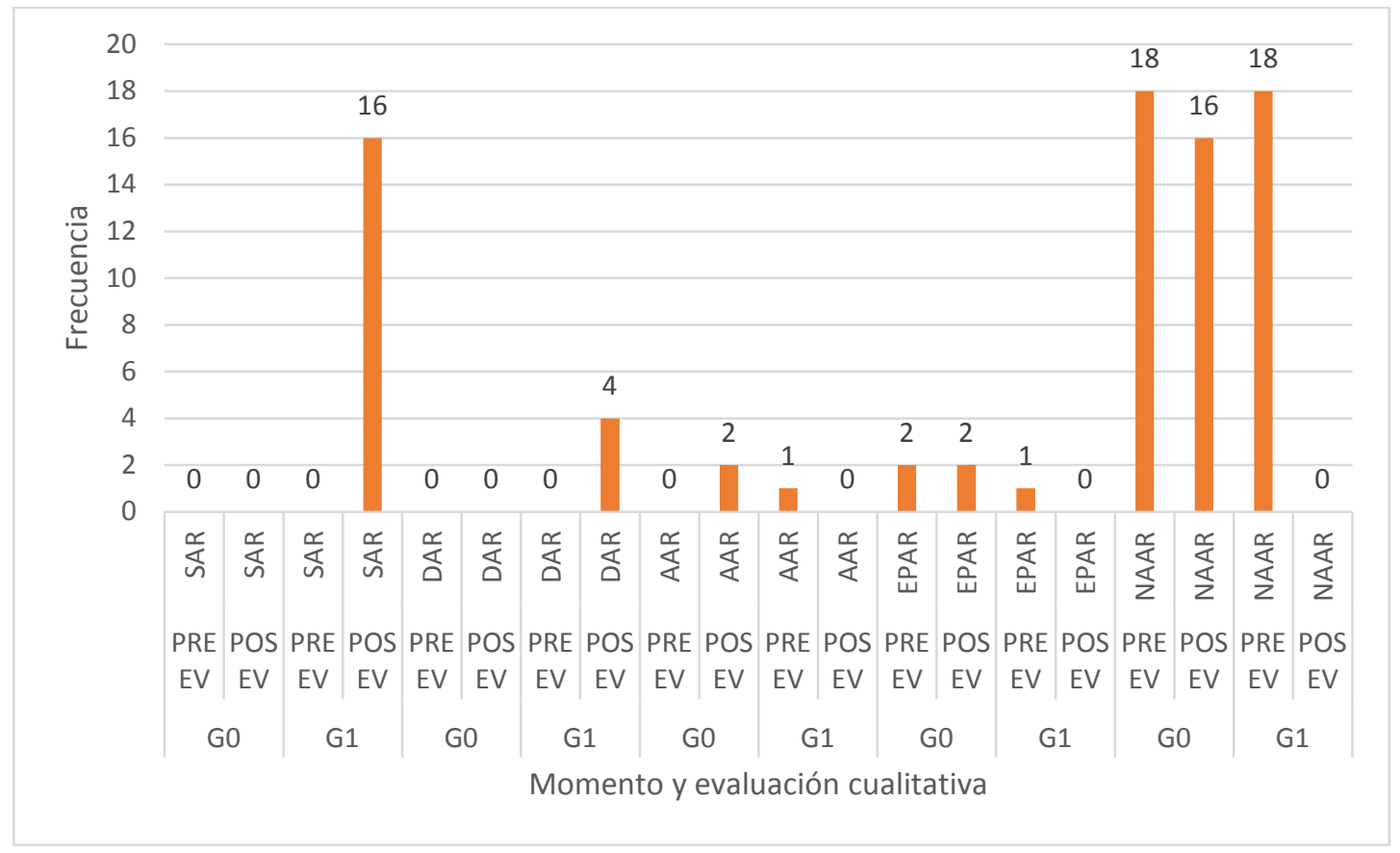

Diseño: equipo de investigación

Fuente: sujetos de estudio

Entrevista a docentes y directora.

Los docentes y directora consideran que la lectura crítica es un proceso donde los estudiantes aplican las estrategias y actividades dadas por los docentes en todos los niveles, esta, permite al estudiante desarrollar el pensamiento crítico; una de las principales estrategias para que se genere con agrado la clase de lectura es la motivación del docente en el aula y por supuesto, del padre de familia en casa; consideran que el aprendizaje es a largo plazo y que es a través de la lectura que los estudiantes relacionan el contexto social y educativo, el estudiante forma su escenario de aprendizaje crítico, motivado e interesante; manifiestan que el aprendizaje de conceptos, palabras, proposiciones e ideas inferidas de la lectura (escogida de la guía del currículo), se deben ajustar a la realidad creando expectativas en los estudiantes, finalizan indicando que el uso de guías pedagógicas son un buen 
Lenin B. Mendieta-Toledo; Oscar A. Garey-Atiencia.

complemento didáctico para el docente y con estos se logra una mejora en la transposición didáctica del objeto del conocimiento, lográndose con esto, un aprendizaje significativamente, haciendo más dinámicas y divertidas las clases, creando un ambiente motivado, activo y participativo, utilizando el conocimiento adquirido en otras asignaturas y en el medio social y cultural en donde se desenvuelven.

\section{Conclusiones.}

Algunos autores que han estudiado las variables de lectura crítica y aprendizaje significativo (Mosquera, 2017; Nivelo \& Quijije, 2017; Cornejo \& Cuzco, 2017) determian la incidencia de la lectura crítica en el aprendizaje significativo de los estudiantes, en las pruebas de lectura crítica realizadas a los estudiantes y el consolidado de calificaciones, se puede evidenciar, que el presente estudio, coincide con los resultados de los autores mencionados. Al igual que Roca (2013) se enfatiza que la lectura crítica debe estar presente en todo proceso formativo, ya que su ausencia genera deficiencia en el desarrollo de aprendizajes significativos en los estudiantes

Remolina (2013) manifiesta que leer significa comprender o interpretar un texto de una u otra forma, dependiendo de los requisitos previos con los que cuente el sujeto lector. El acto de leer posee categorías conceptuales como la operacionalidad cognitiva, el código representacional, y, la modalidad interpretativa, en las pruebas de lectura realizada a los estudiantes del estudio, se pudo determinar que los que pertenecían al G1 comprendían lo que leían, eran capaces de interpretar los textos, no así los estudiantes del G0 que se mantuvieron en la escala de NAAR.

Newman (1990) indica que hay que enseñar en base a la lectura crítica ya que esta condice al cultivo del pensamiento crítico o de orden superior, en el presente estudio se trabajó en base a la lectura crítica y se tomó en cuenta lo señalado por Lipman (1997) quien señala que "es necesario propiciar el 
ejercicio del análisis crítico desde los primeros niveles educativos y (Citados en Díaz, Bar, \& Ortíz, 2015, p. 141).

Se coincide con (Ballester, 2002; Rodríguez, 2008; Díaz, 2010) quienes manifiestan que el aprendizaje es la reestructuración activa de las percepciones, ideas, conceptos y esquemas que poseen los estudiante, de igual forma, en el programa se pudo establecer que los estudiantes logran aprendizajes significativos, cuando son expuestos a la cultura que les rodea, sin olvidar que este aprendizaje se realiza de forma individual e íntimo y va de acuerdo a los requisitos previos que posee el estudiante, tal como lo manifiesta Ausubel (1973) “el aprendizaje significativo es la vía por la que las personas asimilan la cultura que les rodea" (Citado por Pozo, 2006, p. 215).

En las pruebas de lectura realizada a los estudiantes de los grupos control y programa se puede evidenciar en todos los indicadores que se evaluaron, como el G1 mejora en cada uno de ellos, pasando de NAAR a DAR en todos los criterios, esto permite llegar a la conclusión que la lectura crítica incide en el aprendizaje significativo de los estudiantes del subnivel básica media y que los docentes están conscientes que los estudiantes son el centro del aprendizaje.

\section{Bibliografía.}

Acosta, S., \& García , M. (2012). Estrategias de enseñanza utilizadas por los docentes de biología en las universidades públicas. OMNIA, 18(2), 67-82. doi:ISSN: 1315-8856

Alvarado Narváez, P. A. (2013). Incidencia de la lectura comprensiva en el aprendizaje de los estudiantes de 10 año de educación básica, sección séptima del Colegio Nacional Experimental Amazonas de Quito. Universidad Central del Ecuador, Quito. Recuperado el 12 de julio de 2018, de http://www.dspace.uce.edu.ec/handle/25000/3336 
Ballester Vallori, A. (2002). El aprendizaje significativo en el aula. (A. Ballester Vallori, Trad.) España. Recuperado el 2 de enero de 2018, de www.aprendizajesignificativo.es/mats/El_aprendizaje_significativo_en_la_practica.pdf

Cornejo Plaza, N. K., \& Cuzco Cumbe, G. I. (2017). Influencia de la lectura crítica en la calidad del aprendizaje significativo en los estudiantes de quinto grado de educación general básica de la Escuela Fiscal Gabriel García Moreno, zona 6, distrito 01D03, Provincia del Azuay, cantón Santa Isabel, parro. Universidad de Guayaquil, Facultad de Filosofíía, Letras y Ciencias de la Educación. Guayaquil: Universidad de Guayaquil. Facultad de Filosofiía, Letras y Ciencias de la Educación. Obtenido de https://goo.gl/7p4Q13

Díaz, Á. (2010). Estrategias docentes para un aprendizaje significativo. México: Mc Graw Hill. doi:9786071502933

Díaz, J. P., Bar, A., \& Ortíz, M. (oct/dic de 2015). La lectura crítica y su relación con la formación disciplinar de estudiantes universitarios. Revista de la educación superior, 44176, 139-158. doi:ISSN 0185-2760

Mosquera Mora, A. d. (2017). INFLUENCIA DE LA LECTURA CRÍTICA EN LA CALIDAD DEL APRENDIZAJE SIGNIFICATIVO, EN EL ÁREA DE LENGUA Y LITERATURA, EN LOS ESTUDIANTES DEL SÉPTIMO GRADO. Universidad de Guayaquil, Facultad de Filosofía, Letras y Ciencias de la Educación. Guayquil: Unversidad de Guayaquil. Obtenido de https://goo.gl/NX9KDC

Nivelo Moncada, M. M., \& Quijije Alvarado, J. V. (2017). Influencia de la lectura crítica en la calidad del aprendizaje significativo en el área de Lengua y Literatura en los estudiantes del quinto año de educación general básica de la Unidad Educativa Dr Emilio Uzcátegui García, zona 8, distrito 09D07, Provinc. Universidad de Guayaquil, Facultad de Filosofíía, Letras y Ciencias de la Educación. Guayaquil: Universidad de Guayaquil. Facultad de Filosofíía, Letras y Ciencias de la Educación. Obtenido de https://goo.gl/F3hMCF

Pérez Abril, M., \& Roa Casas, C. (2014). Aproximaciones a los instrumentos de la prueba PISALECTURA. Pedagogía y Saberes, 41, 23-35. doi:2500-6436 
Pozo, J. (2006). Teorías cognitivas del aprendizaje (novena ed.). Madrid, España: Morata. doi:ISBN: 13:978-84-7112-335-0

Ramírez, E. (2009). ¿Qué es leer? ¿Qué es la lectura? Investigación bibliotecológica, 23(47), 161188. doi:ISSN 2448-8321

Remolina Caviedes, J. F. (2013). La lectura en Paulo Freire y la competencia lectora de PISA. Educacao, 36(2), 223-231. doi:ISSN: 0101-465X

Roca Llobet, J. (2013). El desarrollo del Pensamiento Crítico a través de diferentes metodologías docentes en el Grado en Enfermería. Barcelona: Universidad Autónoma de Barcelona. doi:9788449041587

Rodríguez Palmero, M. L. (2008). La teoría del aprendizaje significativo en la perspectiva de la psicología educativa. Barcelona, España: Octaedro. doi:ISBN: 978-84-8063-290-4

Solé Gallart, I. (2009). Estrategias de lectura (21 ed.). Barcelona: GRAO. doi:978-84-7827-209-9

White, H., Sabarwal , S., \& de Hoop, T. (2014). Síntesis metodológica $n .^{\circ}$ 7: Ensayos controlados aleatorios. Florencia: Centro de Investigaciones Innocenti de UNICEF. Obtenido de https://goo.gl/Ef2e7S 